\title{
Overexpression of CHAF1A correlates with poor prognosis and tumor immunosuppressive microenvironment and resistance to treatment in cancer
}

Sifeng Qu ( $\sim$ qusifeng@sdu.edu.cn )

Shandong University

Xia Sun

Shandong University

Qiushuang Ma

Shandong University

Yahong Cheng

Shandong University

Huangwei Huang

Shandong University

Jing Qin

Shandong University

\section{Research Article}

Keywords: CHAF1A, cancer, prognosis, metabolism, immune

Posted Date: February 23rd, 2022

DOl: https://doi.org/10.21203/rs.3.rs-1366052/v1

License: (c) (1) This work is licensed under a Creative Commons Attribution 4.0 International License.

Read Full License 


\section{Abstract}

Background: As distinct marker of proliferating cells, chromatin assembly factor-1 (CAF-1) was critical in DNA replication. CHAF1A, the major subunit in CAF-1 complex, has been shown to be highly expressed in some cancer types. However, there is paucity information about the clinical significance, molecule functions and co-expressed gene network of CHAF1A in breast cancer.

Methods: Bioinformatic analysis of CHAF1A and its co-expression gene network were performed using various public databases.

Results: Abnormally high expression of CHAF1A was found in 20 types of cancer tissues. Elevated expression of CHAF1A was positively correlated with breast cancer progression and poor patient outcome. Co-expression gene network analysis indicated that CHAF1A was associated with not only cell proliferation, DNA repair, apoptosis, but also cancer metabolism, immune system, drug resistance and so on. More importantly, higher expression of CHAF1A was positively correlated with immunosuppressive microenvironment and resistance to endocrine therapy and chemotherapy.

Conclusions: The current study indicates that elevated expression of CHAF1A can be used as diagnosis and poor prognosis biomarker of breast cancer. Although higher expression of CHAF1A induced fast resistance to endocrine therapy and chemotherapy, it may be a potential therapeutic target and biomarker to predict the sensitivity of immunotherapy in breast cancer patients.

\section{Introduction}

Cancer is the leading cause of death and a major threaten to public health worldwide [1]. Cancer cell proliferation is the fundamental precondition for disease development and progression. Among the proteins involved in the assembly of DNA into chromatin, the chromatin assembly factor-1 (CAF-1) plays important role in chromatin assembly and histone proteins deposition on to the DNA [2]. CAF-1, an outstanding marker of proliferating cell [3], has been found to be the key regulator in DNA replication and chromatin restoration [4]. The expression of CAF-1 is positively correlated with the expression of Ki-67 in cancers [5].

CAF-1 is a nuclear complex containing three subunits in human cells, CHAF1A (p150), CHAF1B (p60), and RBBP4 (p48) [5]. Among the three subunits, CHAF1A is the major one and plays essential role in CAF-1 complex $[6,7]$. It is shown that cells were not in S phase when Chaf1 a failed to bind to mouse heterochromatin-binding protein-1 (Hp1) during mitosis [8]. However, homozygous deletion of Chaf1a in mice was fatal to mice embryos. And absence of Chaf1a in these embryos led to alterations in the nuclear organization of constitutive heterochromatin [9]. In recent years, studies have shown that the abnormally high expression of CHAF1A is closely related to the development of some kinds of cancer types, such as neuroblastoma, lung cancer, ovarian cancer, and gastric cancer $[4,6,10,11]$. Therefore, CHAF1A is considered to be one of the important oncogenic factors. However, CHAF1A has rarely been reported in breast cancer. And the precise mechanism of breast cancer development and progression 
remained unclear. Therefore, it was crucial to find a novel and reliable biomarker for diagnosis and prediction of prognosis and treatment response in breast cancer.

In the current study, we took a comprehensive approach in investigating the genomic alterations of CHAF1A and demonstrating CHAF1A expression profiles in various cancer types. Our study not only confirmed CHAF1A abnormally high expression in cancers especially in breast cancer, but also demonstrated a strong correlation between CHAF1A overexpression, breast cancer molecular subtype, prognosis and treatment response. Co-expression network analysis was conducted to further explore the underlying roles of CHAF1A. Tumor immunosuppressive microenvironment was also explored to find out the association between CHAF1 A and immune cells. Here, we provide evidence to demonstrate that CHAF1A could be served as a potential biomarker for breast cancer diagnosis, prognosis, sensitivity of immunotherapy and target of therapeutics.

\section{Materials And Methods}

\section{Catalogue of Somatic Mutations in Cancer (COSMIC) database analysis}

The COSMIC database [12] is a comprehensive resource for exploring somatic mutations in human cancer. The latest version was released on May 28, 2021 (v94), which included gene mutations, gene fusions, genomic rearrangements, and copy number variations across 1,491,089 cancer samples. As such, genomic alterations of CHAF1A were summarized using COSMIC database.

\section{Assessment of CHAF1A expression from Oncomine database}

Oncomine [13] is a cancer database with genome-wide expression analyses of 715 datasets and a total of 12764 normal and 86733 tumor samples. Through "differential analysis" module, the expression of a single gene could be analyzed across different types of cancers compared with corresponding normal tissues.

\section{TIMER 2.0}

TIMER 2.0 [14-16] is a resource for comprehensive analysis of cancer infiltrating immune cells in a large variety of malignant diseases. There are three major modules for investigation of immune association, cancer exploration and immune estimation, including gene expression, gene correlation, immune infiltration in this study. Abbreviations: ACC: Adrenocortical Carcinoma; BLCA: Bladder Urothelial Carcinoma; BRCA: Breast Invasive Carcinoma; CESC: Cervical and Endocervical Cancer; CHOL: Cholangiocarcinoma; COAD: Colon Adenocarcinoma; DLBC: Diffuse Large B-cell Lymphoma; ESCA: 
Esophageal Carcinoma; GBM: Glioblastoma Multiforme; HNSC: Head and Neck Cancer; KICH: Kidney Chromophobe; KIRC: Kidney Renal Clear Cell Carcinoma; KIRP: Kidney Renal Papillary Cell Carcinoma; LAML: Acute Myeloid Leukemia; LGG: Lower Grade Glioma; LIHC: Liver Hepatocellular Carcinoma; LUAD: Lung Adenocarcinoma; LUSC: Lung Squamous Cell Carcinoma; MESO: Mesothelioma; OV: Ovarian Serous Cystadenocarcinoma; PAAD: Pancreatic Adenocarcinoma; PCPG: Pheochromocytoma and Paraganglioma; PRAD: Prostate Adenocarcinoma; READ: Rectum Adenocarcinoma; SARC: Sarcoma; SKCM: Skin Cutaneous Melanoma; STAD: Stomach Adenocarcinoma; TGCT: Testicular Germ Cell Tumors; THCA: Thyroid Carcinoma; THYM: Thymoma; UCEC: Uterine Corpus Endometrial Carcinoma; UCS: Uterine Carcinosarcoma; UVM: Uveal Melanoma.

\section{UALCAN}

UALCAN [17-19] is an integrated data-mining platform to facilitate the comprehensive analysis of gene expression and protein expression profile across various cancer types based on publicly available cancer OMICS data $\triangle T C G A \triangle C P T A C$ and CBTTC $\bowtie$. It provides patient survival information for protein-coding, miRNA-coding and lincRNA-coding genes at the same time, which could discover candidate proteins that may be used as tumor biomarkers.

\section{Kaplan-Meier Plotter analysis}

The Kaplan-Meier plotter $[20,21]$ is an on-line database which contains the expression of 30,000 genes and the survival data over 25,000 patients from 21 cancer types. The correlation of CHAF1A expression (Probe ID: 214426_X_at, Jetset best probe) and patients' survival were investigated using the log-rank test. The hazard ratio (HR) with $95 \%$ confidence intervals and log-rank $p$ values were noted.

\section{cBio Cancer Genomics Portal (cBioPortal) analysis}

The cBioPortal $[22,23]$ is an open source which provides a comprehensive platform for exploration, interactive visualization and analysis of large-scale cancer genomic datasets for scientific research. The co-expression data of CHAF1A were downloaded in cBioportal database, which was used for further investigation.

\section{OmicShare online platform}

OmicShare $[24,25]$ is a platform for comprehensive data analysis. It contains multiple modules for different uses, such as heatmap, pathway enrichment, Gene Ontology (GO) enrichment, senior bubble plot and so on. 


\section{Gene Set Enrichment Analysis (GSEA)}

GSEA $[26,27]$ is a computational method that determines whether an a priori defined set of genes shows statistically significant, concordant differences between two biological states (e.g. phenotypes). The enrichment of CHAF1A co-expression genes were used to investigate potential functions and mechanisms of CHAF1A. Normalized enrichment score (NES) was calculated, and nominal $p$ value $<0.05$ was considered as significant enrichment terms.

\section{Gene Expression Profiling Interactive Analysis (GEPIA)}

GEPIA [28] contains 9,736 tumors and 8,587 normal samples from TCGA and Genotype-Tissue Expression (GTEx) projects. It is used for the pan-cancer analysis of the RNA-sequencing expression data, including gene expression, gene correlation, survival rate and so on.

\section{Statistical analysis}

Unless otherwise mentioned, all of these analyses were conducted with $p<0.05$ as the significance threshold. ANOVA was used to analyze the expression of CHAF1A in GEPIA database. Log-rank $p$ value was used for Kaplan-Meier Plotter analysis, and nominal $p$ value was used for GSEA analysis. The data in Oncomine and UALCAN were analyzed by students' t-test. Spearman's Correlation was used for selection of co-expression genes in cBioportal database and TIMER database analysis. When $|\mathrm{Rho}|>0.1$, it indicated that there was a correlation between the genes and immune cells.

\section{Results}

\section{Genomic Alterations of CHAF1A}

In order to identify the contribution of CHAF1A gene in human cancers, COSMIC (v94 GRCh38) [12] was applied for genomic alteration assessment. In the latest released version of COSMIC, CHAF1A was tested in 39,615 cancer samples across 40 different types of cancer. The total mutation frequency of CHAF1A was $1.105 \%$, while missense mutation counted for $0.792 \%$ and the rest were nonsense and synonymous mutations (Table 1). Compared with mutation, Insertion and Deletion were rarely found in cancers. Only 1 Frameshift Insertion (0.003\%), 3 Inframe Deletion (0.008\%) and 5 Frameshift Deletion $(0.012 \%)$ were found in cancers (Table 1 ). The total frequency of copy number alterations was $0.089 \%$ in cancers, including $0.008 \%$ for copy number gain while $0.081 \%$ for copy number loss (Table 1 ). As shown in Supplementary Table 1, one copy number gain was found in malignancies of central nervous system, haematopoietic and lymphoid, and lung, respectively. Copy number loss was found in 12 malignancies, with the highest rate of $1.154 \%$ in upper aerodigestive tract. Taken together, the results indicated that there were no major alterations in the sequence or copy number of the CHAF1A gene that could be responsible for the development of cancers. 
Table 1

Genetic alterations affecting CHAF1A in 39,615 cancer samples (COSMIC database)

\begin{tabular}{|lll|}
\hline Genetic Alteration & Number & Percentage \\
\hline Substitution Nonsense & 14 & $0.035 \%$ \\
\hline Substitution Missense & 314 & $0.792 \%$ \\
\hline Substitution Synonymous & 110 & $0.277 \%$ \\
\hline Inframe Insertion & 0 & 0 \\
\hline Frameshift Insertion & 1 & $0.003 \%$ \\
\hline Inframe Deletion & 3 & $0.008 \%$ \\
\hline Frameshift Deletion & 5 & $0.012 \%$ \\
\hline Complex Mutation & 0 & 0 \\
\hline Others & 14 & $0.035 \%$ \\
\hline Copy Number Gain & 3 & $0.008 \%$ \\
\hline Copy Number Loss & 32 & $0.081 \%$ \\
\hline
\end{tabular}

\section{Elevated CHAF1A expression in human cancers}

Using TIMER 2.0 database $[14,15]$, the gene expression of CHAF1A was investigated in various cancer types. Among the 22 paired tumor and adjacent normal tissues, the expression of CHAF1A was significantly elevated in 20 types of malignancies compared with normal tissues (Figure 1). In consistent with the result from TIMER 2.0 database, it was also found abnormally high expression of CHAF1A in 36 study cohorts covering 17 types of cancers in Oncomine database [13] (Supplementary Table 2). In both two databases, breast cancer cohort contains the greatest amount of patient samples. As well, the $p$ value of CHAF1A expression between cancer tissues and normal tissues was the most significant in breast cancer cohort. Thereby, we focused on breast cancer in the further investigation.

\section{Correlation of CHAF1A expression with breast cancer phenotype and prognosis}

To further investigate the clinical significance and application of CHAF1A in breast cancer, we examined the correlation between CHAF1A expression and breast cancer phenotype in UALCAN database [17-19]. Both mRNA expression and protein expression of CHAF1A were significantly higher in breast cancer tissues compared with normal tissues (Figure 2A, B). Furthermore, among the subtypes of breast cancer, 
the expression of CHAF1A was higher in triple negative breast cancer (TNBC) compared with either luminal or HER2 positive breast cancers in both mRNA level and protein level (Figure 2C, D). In addition, the expression of CHAF1A was higher in TP53 mutant breast cancer tissues compared with TP53 wild type breast cancer tissues (Figure 2E).

Then, we determined the effect of elevated CHAF1A expression on patients' outcome. Elevated expression of CHAF1A was significantly correlated with lower overall survival (Figure 2F), shorter recurrence free survival (Figure 2G), and shorter distant metastasis free survival (Figure $2 \mathrm{H}$ ) of breast cancer patients obtained from Kaplan-Meier plotter [20] survival analysis. The HR was 1.33, 1.25, and 1.48, respectively. Taken together, the data indicate that elevated expression of CHAF1A is a prognostic biomarker of poor clinical outcome in breast cancer patients. The mechanisms underlying these events need to be further investigated.

\section{Gene co-expression network of CHAF1A and its potential regulatory mechanisms}

Gene co-expression networks currently have been frequently used for exploration for the functional roles of the target genes [29]. In cBioPortal database [22, 23], the co-expression genes of CHAF1A in breast invasive carcinoma were downloaded, including three cohorts of TCGA data: Nature 2021, Cell 2015, and Firehose Legacy [30, 31]. In order to get more precision co-expression networks, the genes shown consistent positive correlation score and consistent negative correlation score in all three cohorts were selected for further investigation.

The KEGG pathway annotation and enrichment of co-expression genes were generated with OmicShare online platform [24,25]. Figure 3 displayed the comprehensive functions and mechanisms of CHAF1A with statistical significance. The major functional roles of CHAF1A and its co-expressed genes focused on metabolism, genetic information processing, environmental information processing, cellular processes, organismal systems and human diseases (Figure 3A). Especially, the effect on amino acid metabolism, energy metabolism, immune system, and drug resistance are more closely connected with potential response to cancer treatment. Among the top 20 KEGG pathway enrichment (Figure 3B), the coexpression network was covered cancer metabolism, stemness, microRNA, cell cycle, RNA splicing, TGF- $\beta$ pathway, apoptosis and DNA repair.

In addition, the pathways involved in cancer hallmarks were investigated using GSEA [26, 27]. As shown in Figure 3C, the co-expression network in TCGA breast cancer cohorts was significantly positively enriched in E2F targets, G2M checkpoint, mitotic spindle, DNA repair, MYC target, MTORC1 signaling, and glycolysis. The results from GSEA analysis were specific to signaling pathways, while still consistent with that from OmicShare KEGG pathway annotation and enrichment. Interestingly, cancer metabolism was of the most outstanding generated by various analyses, which suggested that CHAF1A might be critical to 
the development and progression of breast cancer, thus might provide novel target for the treatment of breast cancer.

\section{Association of elevated CHAF1A expression with immunosuppressive tumor microenvironment}

Since the co-expression network analysis indicated that immune system might be involved (Figure 3A), we looked deep into the tumor-infiltrating immune cells of breast cancer samples to investigate the correlation between CHAF1A and immune response in TIMER 2.0 database [14-16]. In breast cancer, it was found that the expression of CHAF1A was negatively correlated with CD8+ T cell, but positively correlated with regulatory T cell (Treg) and MDSC (Myeloid-derived suppressor cell) (Figure 4A).

Furthermore, we checked the correlation between CHAF1A and immune checkpoints in TIMER 2.0 database. The expression of CHAF1A was positively correlated with that of both CD274 and CTLA4 (Figure 4B). This correlation between CHAF1A and either CD274 or CTLA4 was also confirmed by GEPIA database (Figure 4C). Thereby, the elevated expression of CHAF1A might be related with suppressive tumor microenvironment resulting in poor survival outcome. However, these patients might response to immunotherapy.

\section{Elevated CHAF1A expression as a potential predictive biomarker to therapeutic treatment}

In order to investigate whether elevated CHAF1A expression detected in breast cancer tissues could be used to predict the sensitivity of the patients to therapeutic treatment, the correlations between the oncolytic response of patients and elevated CHAF1A expression were determined in Kaplan-Meier plotter [20].

It was found that the breast cancer patients with elevated expression of CHAF1A showed significantly shorter recurrence free survival whether receiving endocrine therapy or chemotherapy (Figure 5A). Since the mechanisms of endocrine therapy and chemotherapy are different, the breast cancer patients were divided into two groups, one group received endocrine therapy and the other group received chemotherapy. Patients with elevated expression of CHAF1A showed significantly shorter recurrence free survival in each group (Figure 5B and C). As such, breast cancer patients with higher expression of CHAF1A might progress into treatment resistance to endocrine therapy and chemotherapy in shorter time.

\section{Discussion}

During the past decades, therapeutic options in cancers have been fast developed. The current treatment paradigm is now focused on mechanism-based therapeutics with selectivity, such as targeted therapy and immunotherapy [32]. However, neither targeted therapy or immunotherapy can reach effective and 
durable results. Increasing evidences indicate that some targeted therapy can promote the anti-cancer immune response. As such, the combined application of targeted therapy and immunotherapy may generate synergistic anti-cancer efficacy for patients [33]. Thereby, the screening of immune-related therapeutic target is of great interests in cancer.

In the current study, we have reported that CHAF1A was highly expressed in 20 types of cancers. To rule out the potential effect of genomic alterations of CHAF1A in cancers, mutation, insertion, deletion and copy number alterations were investigated in COSMIC and Oncomine. The frequency of genetic alterations was too low to lead cancers development and progression. Elevated expression of CHAF1A has been reported to be associated with several solid cancers $[4,6,7,11,34]$, it was found that this phenomenon was commonly shared in more cancer types, indicating that abnormal higher expression of CHAF1A could be a potential biomarker for cancer diagnosis. Moreover, there was no study about the functional roles and mechanisms of CHAF1A in breast cancer. Thereby, in the current study, we have shown the comprehensive profile of CHAF1A in breast cancer.

As a hormone related cancer type, the classification of breast cancer is based on the expression of estrogen receptor (ER), progesterone receptor (PR) and human epidermal growth factor receptor 2 (HER2). TNBC is characterized by lack of expression of ER, PR and HER2 [35]. More importantly, TNBC is the most aggressive subtype of breast cancer [36]. The expression of CHAF1A was higher in breast cancer tissues than benign tissues. Furthermore, the expression of CHAF1A was significantly higher in TNBC than either luminal or HER2 positive breast cancer. In addition, it was found that the expression of CHAF1A was higher in TP53 mutant breast cancer patients. Since it was reported that the frequency of TP53 mutation was high in TNBC [30], it was consistent with our finding that the expression of CHAF1A was not only higher in TP53 mutant breast cancer, but also higher in TNBC. In addition, we found statistically significant correlation between elevated CHAF1A expression and poor outcome of breast cancer patients. The data indicate that the elevated expression of CHAF1A may be a poor prognostic biomarker in breast cancer.

To identify the potential mechanisms of elevated expression of CHAF1A in breast cancer, the bioinformatics analysis was applied using CHAF1A co-expression network. The major biological effects and signaling pathways demonstrated that CHAF1A played important roles in breast cancer progression. Some of these functions and mechanisms of CHAF1A were reported in other cancer types, however, most of these were shown in the first time. In ovarian cancer, it was found that CHAF1A was involved in DNA repair, apoptosis, and cell cycle [11], which were confirmed in our study by pathway enrichment as shown in KEGG pathways (such as cell cycle, p53 signaling pathway, DNA replication and mismatch repair) and GSEA enrichment (such as E2F targets, G2M checkpoint, mitotic spindle and DNA repair). It was reported that CHAF1A could bind to the promoter of c-Myc to enhance the transcriptional expression of c-Myc in gastric cancer [4], which was consistent with the current finding that CHAF1A was positively correlated with Myc targets. These findings indicated that CHAF1A played essential role to promote breast cancer growth. As such, CHAF1A could be potential therapeutic target in breast cancer. 
More importantly, it was found that CHAF1A could affect cancer metabolism and immune system. In recent years, it has been demonstrated that cancer metabolism was important since it can affect immunotherapy and chemotherapy in cancer treatment [37-40]. It was interesting to find that the elevated expression of CHAF1A was negatively correlated with CD8 + cell, but positively correlated with Treg and MDSC in breast cancer. As well, the expression of CHAF1A was positively correlated with immune checkpoints, i.e. CD274 and CTLA4. It is widely acknowledged that glycolysis is important factor to induce immunosuppressive microenvironment in cancer [41]. In the current study, elevated expression of CHAF1A is positively correlated with glycolysis in breast cancer. Thereby, the data indicate that elevated expression of CHAF1A is associated with immunosuppression in breast cancer. And CHAF1A targeting therapy combined with immune checkpoint inhibitors might be potential therapeutic strategy for breast cancer patients with increased expression of CHAF1A.

Cancer metabolism reprogramming has been found to mediate drug resistance in cancer patients [42]. To investigate the correlation between CHAF1A and the response to treatment, breast cancer patients received either endocrine therapy or chemotherapy were enrolled in Kaplan-Meier plotter. Since we found that $\mathrm{CHAF1A}$ could induce metabolic reprogramming in breast cancer, it was not surprising that patients with increased CHAF1A expression developed drug resistance in a relatively short period of time. This was consistent with the research finding that elevated expression of CHAF1A could promote thymidylate synthetase activity, leading to 5-FU resistance in gastric cancer [43]. In addition, it was reported that abnormally high expression of CHAF1A could affect the metabolic pathways of some amino acids, such as methionine, eventually inducing 5'-methylthioadenosine (MTA) accumulation in neuroblastoma [7]. Homozygous deletion of the methylthioadenosine phosphorylase (MTAP) is frequently found in human cancers, and the application of purine analogue has been shown to be effective therapeutic option in MTAP deletion cancer patients [44]. Since elevated expression of CHAF1A may cause accumulation of MTA via regulating amino acid metabolism, it is possible that purine analogue might be potential treatment option in MTAP deletion breast cancer patient with higher expression of CHAF1A.

In conclusion, the current study indicates that elevated expression of CHAF1A can be used as diagnostic biomarker in a large variety of human cancers. Moreover, elevated expression of CHAF1A is a promising prognostic predictor and a potential biomarker of drug resistance in breast cancer. In addition, it may serve as potential therapeutic target and be used as a biomarker to predict the sensitivity of immunotherapy in breast cancer patients.

\section{Abbreviations}

CAF-1: chromatin assembly factor-1; ACC: Adrenocortical Carcinoma; BLCA: Bladder Urothelial Carcinoma; BRCA: Breast Invasive Carcinoma; CESC: Cervical and Endocervical Cancer; CHOL: Cholangiocarcinoma; COAD: Colon Adenocarcinoma; DLBC: Diffuse Large B-cell Lymphoma; ESCA: Esophageal Carcinoma; GBM: Glioblastoma Multiforme; HNSC: Head and Neck Cancer; KICH: Kidney Chromophobe; KIRC: Kidney Renal Clear Cell Carcinoma; KIRP: Kidney Renal Papillary Cell Carcinoma; LAML: Acute Myeloid Leukemia; LGG: Lower Grade Glioma; LIHC: Liver Hepatocellular Carcinoma; LUAD: 
Lung Adenocarcinoma; LUSC: Lung Squamous Cell Carcinoma; MESO: Mesothelioma; OV: Ovarian Serous Cystadenocarcinoma; PAAD: Pancreatic Adenocarcinoma; PCPG: Pheochromocytoma and Paraganglioma; PRAD: Prostate Adenocarcinoma; READ: Rectum Adenocarcinoma; SARC: Sarcoma; SKCM: Skin Cutaneous Melanoma; STAD: Stomach Adenocarcinoma; TGCT: Testicular Germ Cell Tumors; THCA: Thyroid Carcinoma; THYM: Thymoma; UCEC: Uterine Corpus Endometrial Carcinoma; UCS: Uterine Carcinosarcoma; UVM: Uveal Melanoma; ER: estrogen receptor; PR: progesterone receptor; HER2: human epidermal growth factor receptor 2; TNBC: triple negative breast cancer.

\section{Declarations}

\section{Ethics approval and consent to participate:}

Not applicable.

\section{Consent for publication:}

Not applicable.

\section{Availability of data and material:}

The datasets used and analyzed during the current study are available in the public databases listed in the manuscript.

\section{Competing interests:}

All of the authors declare that they have no conflicts of interest.

\section{Funding:}

This project was supported by the National Natural Science Foundation, China (81872894), Joint Fund of Shandong Provincial Natural Science Foundation (ZR2021LSW026), China Postdoctoral Science Foundation (2020M672066), Shandong Provincial Natural Science Foundation (ZR2020MH264), Beijing Bethune Charitable Foundation (mnzl202016), Shandong Province Postdoctoral Innovation Project (202002010), CSCO-Pilot Cancer Research Foundation (Y-2019AZQN-0039).

\section{Authors' contributions:}

Study concept and design: Sifeng Qu 
Acquisition of data: Xia Sun, Qiushuang Ma, Yahong Cheng, Huangwei Huang, Jing Qin

Analysis and interpretation of data: Sifeng Qu, Xia Sun, Qiushuang Ma, Yahong Cheng, Huangwei Huang, Jing Qin

Draft of the manuscript: Xia Sun, Sifeng Qu

Obtained funding: Xia Sun, Sifeng Qu

All authors read and approved the final manuscript.

\section{References}

1. Sung H, Ferlay J, Siegel RL, Laversanne M, Soerjomataram I, Jemal A, Bray F: Global cancer statistics 2020: GLOBOCAN estimates of incidence and mortality worldwide for 36 cancers in 185 countries. CA Cancer J Clin2021.

2. Shen J, Liu X, Zhou M, Liu H, Xu L, Meng X: CHAF1A overexpression in human retinoblastoma promotes cell proliferation and suppresses apoptosis. J BUON2020, 25(5):2510-2514.

3. Polo SE, Theocharis SE, Klijanienko J, Savignoni A, Asselain B, Vielh P, Almouzni G: Chromatin assembly factor-1, a marker of clinical value to distinguish quiescent from proliferating cells. Cancer Res2004, 64(7):2371-2381.

4. Zheng L, Liang X, Li S, Li T, Shang W, Ma L, Jia X, Shao W, Sun P, Chen Cet al: CHAF1A interacts with TCF4 to promote gastric carcinogenesis via upregulation of C-MYC and CCND1 expression. EBioMedicine2018, 38:69-78.

5. Sykaras AG, Pergaris A, Theocharis S: Challenging, Accurate and Feasible: CAF-1 as a Tumour Proliferation Marker of Diagnostic and Prognostic Value. Cancers (Basel)2021, 13(11).

6. Liu T, Wei J, Jiang C, Wang C, Zhang X, Du Y, Li J, Zhao H: CHAF1A, the largest subunit of the chromatin assembly factor 1 complex, regulates the growth of H1299 human non-small cell lung cancer cells by inducing G0/G1 cell cycle arrest. Exp Ther Med2017, 14(5):4681-4686.

7. Tao L, Moreno-Smith M, Ibarra-García-Padilla R, Milazzo G, Drolet NA, Hernandez BE, Oh YS, Patel I, Kim JJ, Zorman Bet al: CHAF1A Blocks Neuronal Differentiation and Promotes Neuroblastoma Oncogenesis via Metabolic Reprogramming. Adv Sci (Weinh)2021, 8(19):e2005047.

8. Murzina N, Verreault A, Laue E, Stillman B: Heterochromatin dynamics in mouse cells: interaction between chromatin assembly factor 1 and HP1 proteins. Mol Cel/1999, 4(4):529-540.

9. Houlard M, Berlivet S, Probst AV, Quivy JP, Héry P, Almouzni G, Gérard M: CAF-1 is essential for heterochromatin organization in pluripotent embryonic cells. PLoS Genet2006, 2(11):e181.

10. Barbieri E, De Preter K, Capasso M, Chen Z, Hsu DM, Tonini GP, Lefever S, Hicks J, Versteeg R, Pession Aet al: Histone chaperone CHAF1A inhibits differentiation and promotes aggressive neuroblastoma. Cancer Res2014, 74(3):765-774. 
11. Xia D, Yang X, Liu W, Shen F, Pan J, Lin Y, Du N, Sun Y, Xi X: Over-expression of CHAF1A in Epithelial Ovarian Cancer can promote cell proliferation and inhibit cell apoptosis. Biochem Biophys Res Commun2017, 486(1):191-197.

12. Tate JG, Bamford S, Jubb HC, Sondka Z, Beare DM, Bindal N, Boutselakis H, Cole CG, Creatore C, Dawson Eet al: COSMIC: the Catalogue Of Somatic Mutations In Cancer. Nucleic Acids Res2019, 47(D1):D941-D947.

13. Rhodes DR, Kalyana-Sundaram S, Mahavisno V, Varambally R, Yu J, Briggs BB, Barrette TR, Anstet MJ, Kincead-Beal C, Kulkarni Pet al: Oncomine 3.0: genes, pathways, and networks in a collection of 18,000 cancer gene expression profiles. Neoplasia2007, 9(2):166-180.

14. Li T, Fu J, Zeng Z, Cohen D, Li J, Chen Q, Li B, Liu XS: TIMER2.0 for analysis of tumor-infiltrating immune cells. Nucleic Acids Res2020, 48(W1):W509-W514.

15. Li T, Fan J, Wang B, Traugh N, Chen Q, Liu JS, Li B, Liu XS: TIMER: A Web Server for Comprehensive Analysis of Tumor-Infiltrating Immune Cells. Cancer Res2017, 77(21):e108-e110.

16. Li B, Severson E, Pignon JC, Zhao H, Li T, Novak J, Jiang P, Shen H, Aster JC, Rodig Set al: Comprehensive analyses of tumor immunity: implications for cancer immunotherapy. Genome Biol2016, 17(1):174.

17. Chen F, Chandrashekar DS, Scheurer ME, Varambally S, Creighton CJ: Global molecular alterations involving recurrence or progression of pediatric brain tumors. Neoplasia2022, 24(1):22-33.

18. Chen F, Chandrashekar DS, Varambally S, Creighton CJ: Pan-cancer molecular subtypes revealed by mass-spectrometry-based proteomic characterization of more than 500 human cancers. Nat Commun2019, 10(1):5679.

19. Chandrashekar DS, Bashel B, Balasubramanya SAH, Creighton CJ, Ponce-Rodriguez I, Chakravarthi BVSK, Varambally S: UALCAN: A Portal for Facilitating Tumor Subgroup Gene Expression and Survival Analyses. Neoplasia2017, 19(8):649-658.

20. Győrffy B: Survival analysis across the entire transcriptome identifies biomarkers with the highest prognostic power in breast cancer. Comput Struct Biotechnol J2021, 19:4101-4109.

21. Lánczky A, Győrffy B: Web-Based Survival Analysis Tool Tailored for Medical Research (KMplot): Development and Implementation. J Med Internet Res2021, 23(7):e27633.

22. Gao J, Aksoy BA, Dogrusoz U, Dresdner G, Gross B, Sumer SO, Sun Y, Jacobsen A, Sinha R, Larsson Eet al: Integrative analysis of complex cancer genomics and clinical profiles using the cBioPortal. Sci Signa/2013, 6(269):pl1.

23. Cerami E, Gao J, Dogrusoz U, Gross BE, Sumer SO, Aksoy BA, Jacobsen A, Byrne CJ, Heuer ML, Larsson Eet al: The cBio cancer genomics portal: an open platform for exploring multidimensional cancer genomics data. Cancer Discov2012, 2(5):401-404.

24. Su M, Guo C, Liu M, Liang X, Yang B: Therapeutic targets of vitamin C on liver injury and associated biological mechanisms: A study of network pharmacology. Int Immunopharmacol2019, 66:383-387.

25. Wang S, Su W, Zhong C, Yang T, Chen W, Chen G, Liu Z, Wu K, Zhong W, Li Bet al: An Eight-CircRNA Assessment Model for Predicting Biochemical Recurrence in Prostate Cancer. Front Cell Dev 
Bio/2020, 8:599494.

26. Subramanian A, Tamayo P, Mootha VK, Mukherjee S, Ebert BL, Gillette MA, Paulovich A, Pomeroy SL, Golub TR, Lander ESet al: Gene set enrichment analysis: a knowledge-based approach for interpreting genome-wide expression profiles. Proc Natl Acad Sci U S A2005, 102(43):15545-15550.

27. Mootha VK, Lindgren CM, Eriksson KF, Subramanian A, Sihag S, Lehar J, Puigserver P, Carlsson E, Ridderstråle M, Laurila Eet al: PGC-1alpha-responsive genes involved in oxidative phosphorylation are coordinately downregulated in human diabetes. Nat Genet2003, 34(3):267-273.

28. Tang Z, Li C, Kang B, Gao G, Zhang Z: GEPIA: a web server for cancer and normal gene expression profiling and interactive analyses. Nucleic Acids Res2017, 45(W1):W98-W102.

29. Villa-Vialaneix N, Liaubet L, Laurent T, Cherel P, Gamot A, SanCristobal M: The structure of a gene coexpression network reveals biological functions underlying eQTLs. PLoS One2013, 8(4):e60045.

30. Network CGA: Comprehensive molecular portraits of human breast tumours. Nature2012, 490(7418):61-70.

31. Ciriello G, Gatza ML, Beck AH, Wilkerson MD, Rhie SK, Pastore A, Zhang H, McLellan M, Yau C, Kandoth Cet al: Comprehensive Molecular Portraits of Invasive Lobular Breast Cancer. Cel/2015, 163(2):506-519.

32. Vanneman M, Dranoff G: Combining immunotherapy and targeted therapies in cancer treatment. Nat Rev Cancer2012, 12(4):237-251.

33. Bergholz JS, Wang Q, Kabraji S, Zhao JJ: Integrating Immunotherapy and Targeted Therapy in Cancer Treatment: Mechanistic Insights and Clinical Implications. Clin Cancer Res2020, 26(21):55575566 .

34. Xu M, Jia Y, Liu Z, Ding L, Tian R, Gu H, Wang Y, Zhang H, Tu K, Liu Q: Chromatin assembly factor 1 , subunit A (P150) facilitates cell proliferation in human hepatocellular carcinoma. Onco Targets Ther2016, 9:4023-4035.

35. Garrido-Castro AC, Lin NU, Polyak K: Insights into Molecular Classifications of Triple-Negative Breast Cancer: Improving Patient Selection for Treatment. Cancer Discov2019, 9(2):176-198.

36. Ensenyat-Mendez M, Llinàs-Arias P, Orozco JIJ, íñiguez-Muñoz S, Salomon MP, Sesé B, DiNome ML, Marzese DM: Current Triple-Negative Breast Cancer Subtypes: Dissecting the Most Aggressive Form of Breast Cancer. Front Oncol2021, 11:681476.

37. DePeaux K, Delgoffe GM: Metabolic barriers to cancer immunotherapy. Nat Rev Immuno/2021, 21(12):785-797.

38. Bader JE, Voss K, Rathmell JC: Targeting Metabolism to Improve the Tumor Microenvironment for Cancer Immunotherapy. Mol Cel/2020, 78(6):1019-1033.

39. Desbats MA, Giacomini I, Prayer-Galetti T, Montopoli M: Metabolic Plasticity in Chemotherapy Resistance. Front Oncol2020, 10:281.

40. Zaal EA, Berkers CR: The Influence of Metabolism on Drug Response in Cancer. Front Onco/2018, 8:500. 
41. Reinfeld BI, Rathmell WK, Kim TK, Rathmell JC: The therapeutic implications of immunosuppressive tumor aerobic glycolysis. Cell Mol Immuno/2022, 19(1):46-58.

42. Chen X, Chen S, Yu D: Metabolic Reprogramming of Chemoresistant Cancer Cells and the Potential Significance of Metabolic Regulation in the Reversal of Cancer Chemoresistance. Metabolites2020, 10(7).

43. Wang D, Li X, Shen B, Chen X, Shu Y: Histone chaperone CHAF1A impacts the outcome of fluoropyrimidines-based adjuvant therapy in gastric cancer by regulating the expression of thymidylate synthetase. Gene2019, 716:144034.

44. Tang B, Lee HO, An SS, Cai KQ, Kruger WD: pecific Targeting of MTAP-Deleted Tumors with a Combination of 2'-Fluoroadenine and 5'-Methylthioadenosine Cancer Res2018, 78(15):4386-4395.

\section{Figures}

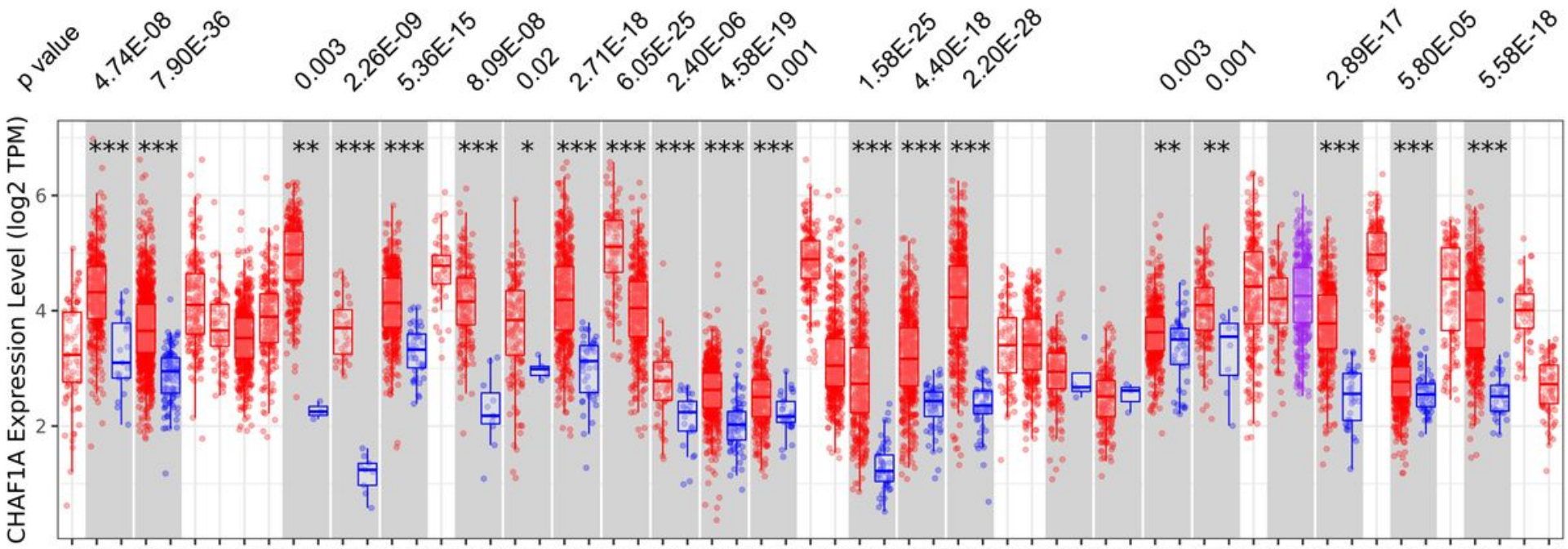

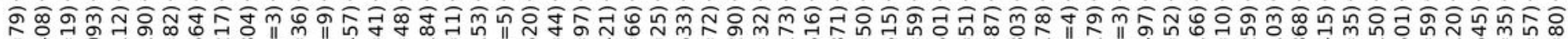

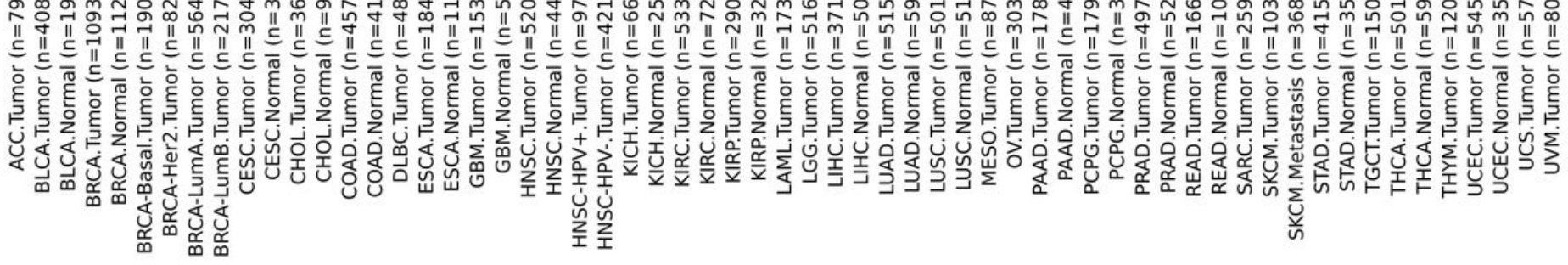

\section{Figure 1}

The expression of CHAF1A is significantly higher in malignant tissues compared with normal tissues in various cancer types.

In TIMER 2.0 database, CHAF1A is significantly highly expressed in 20 types of malignancies compared with normal tissues. The statistical significance was annotated by stars (*: $p<0.05 ; \star \star: p<0.01 ; * \star *$ : $p<0.001)$. 


\section{Figure 2}

\section{Elevated expression of CHAF1A in breast cancer tissues was significantly correlated with poor prognosis of breast cancer patients.}

By using UALCAN database: (A-B) Both mRNA expression and protein expression of CHAF1A were dramatically increased in breast cancer tissues. (C-D) CHAF1A was highly expressed in all subtypes of breast cancer tissues compared with normal tissues. And compared with either Luminal or HER2 positive breast cancers, CHAF1A showed the highest expression in TNBC. (E) The expression of CHAF1A was higher in TP53 mutant breast cancer tissues compared with either TP53 wild type or normal tissues (*ᄎ*: $p<0.001)$. In Kaplan-Meier Plotter survival analysis: $(\mathbf{F})$ The breast cancer patients with relatively higher expression of CHAF1A showed poor overall survival compared to patients with relatively lower CHAF1A expression. (G) The breast cancer patients with relatively higher expression of CHAF1A took less time to develop disease recurrence. $(\mathrm{H})$ The breast cancer patients with higher CHAF1A expression took shorter time to develop distant metastasis.

\section{Figure 3}

\section{Analysis of CHAF1A's functional roles and potential mechanisms.}

(A) The KEGG annotation of CHAF1A co-expression gene network was indicated by OmicShare. The length of the bar stands for the number of genes enriched in each function. (B) The top 20 pathways of CHAF1A co-expression gene network were generated by using OmicShare. The size of node stands for the number of genes enriched in pathway, and the colour stands for $p$ value. (C) The enrichment of cancer hallmarks based on the CHAF1A co-expression gene network using GSEA. 
A

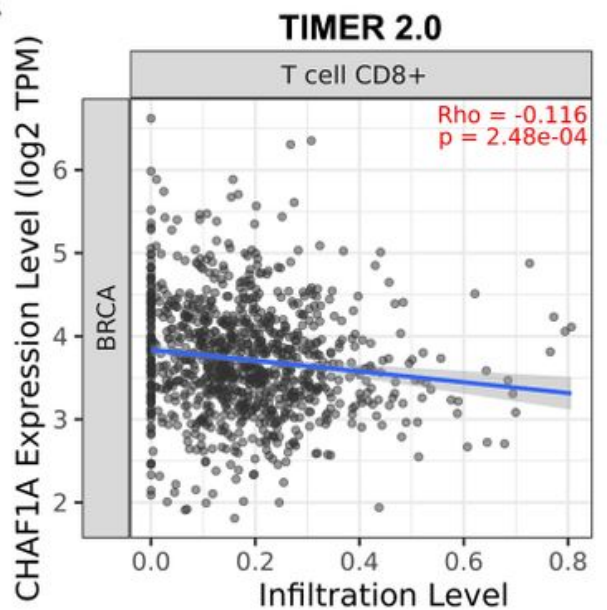

B
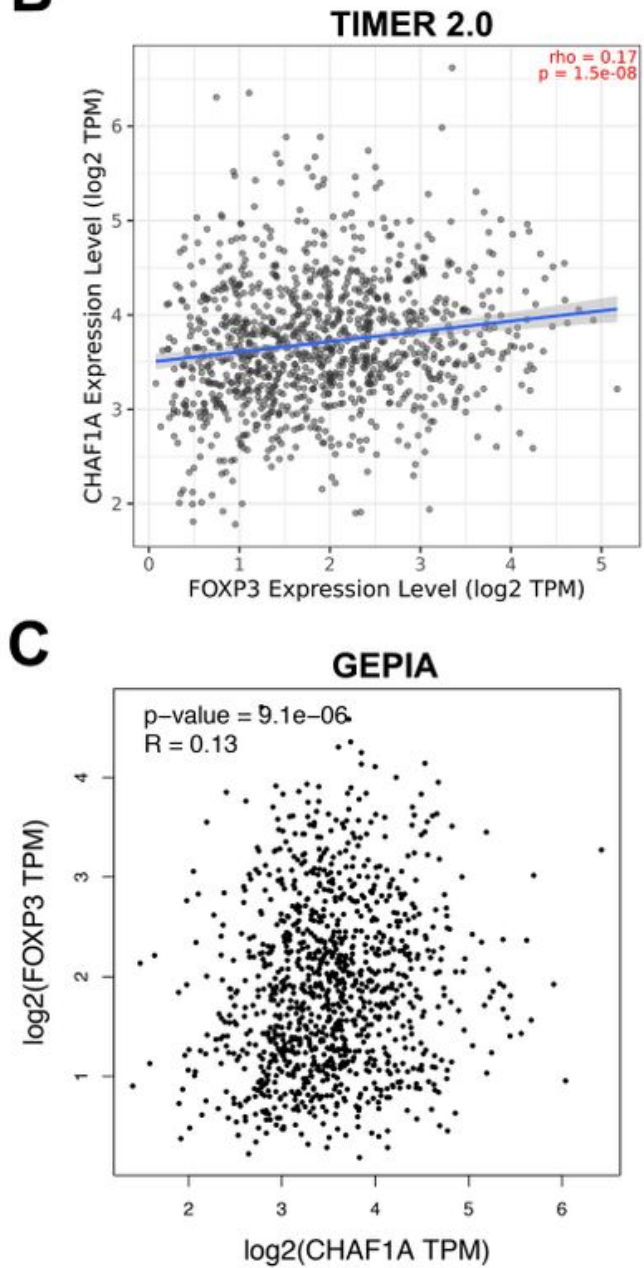

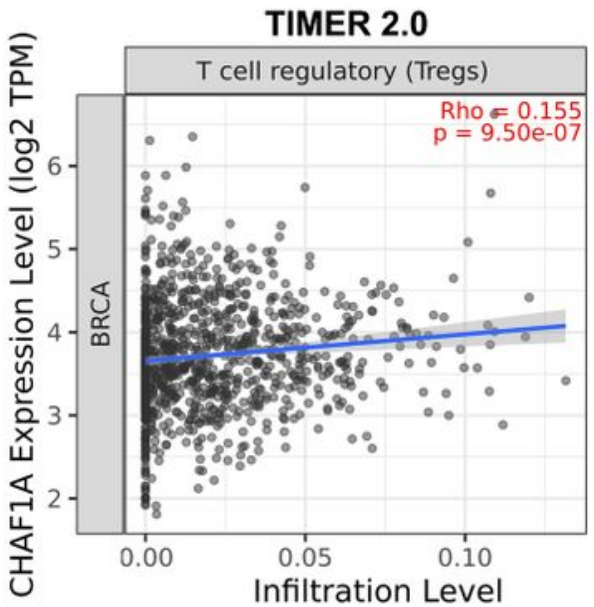

TIMER 2.0
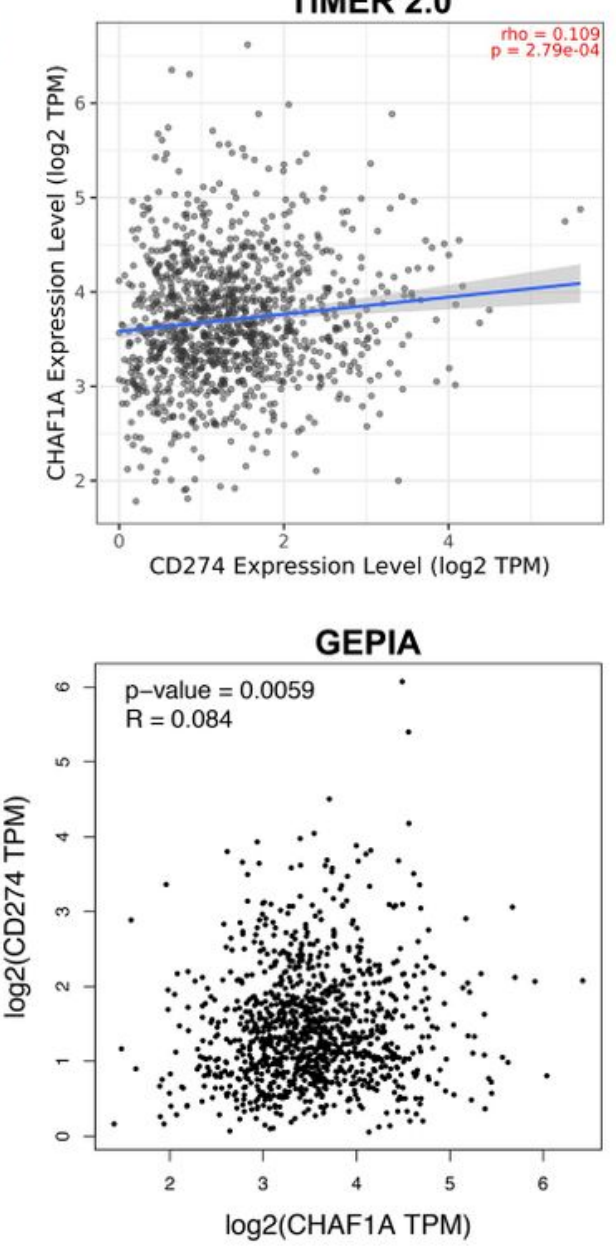

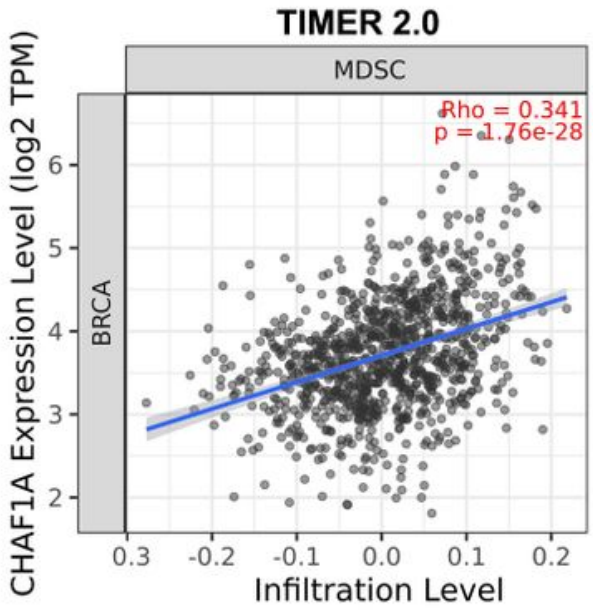

TIMER 2.0
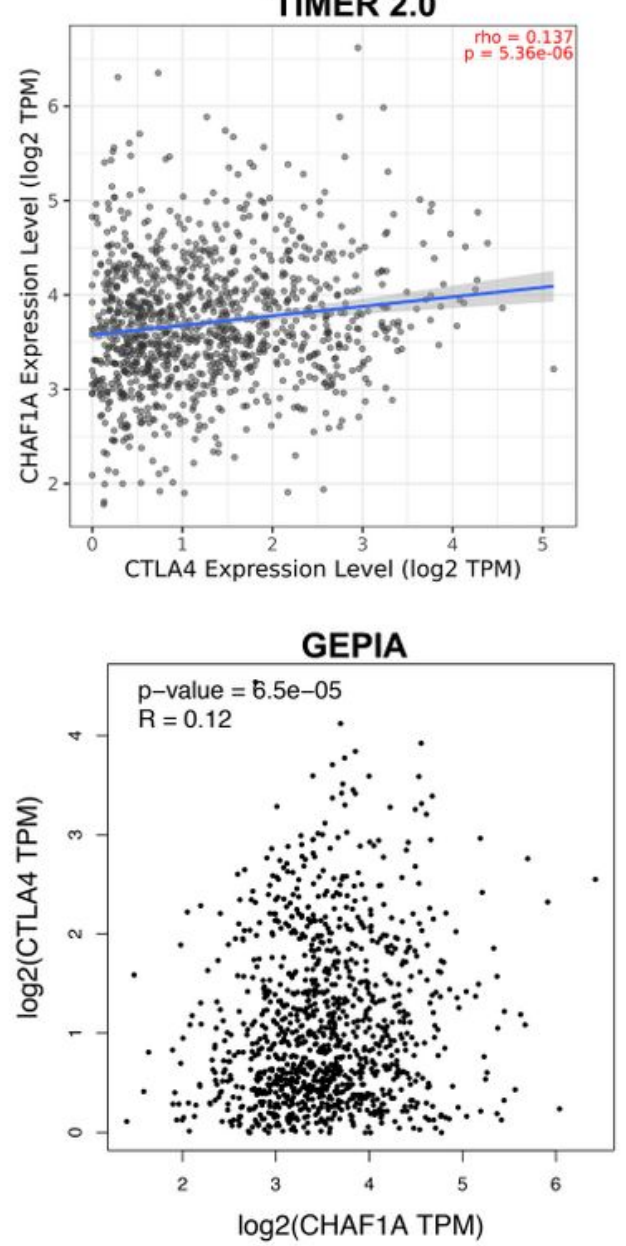

Figure 4

The association of CHAF1A in cancer microenvironment and correlation between CHAF1A and immune cells in breast cancer tissues.

(A) Higher expression of CHAF1A was negatively associated with cancer infiltrating CD8+ T cells, but positively associated with infiltrating regulatory $T$ cells and MDSC cells in breast cancer as indicated in TIMER 2.0 database. (B) The expression of CHAF1A was positively correlated with the expression of 
CD274 and CTLA4 in breast cancer in TIMER 2.0 database. (C) The expression of CHAF1A was positively correlated with the expression of CD274 and CTLA4 in breast cancer in GEPIA database.
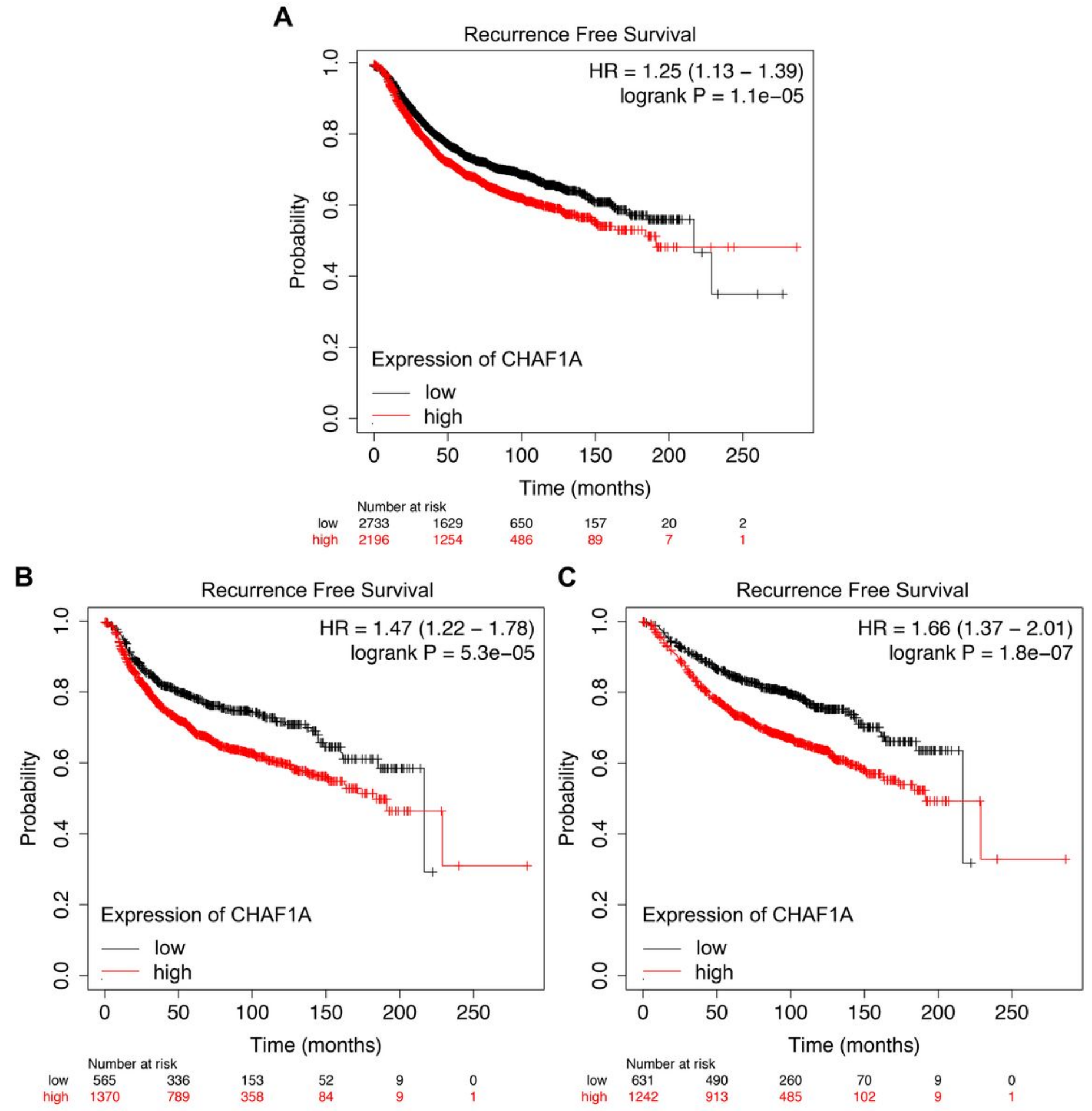

Figure 5

Resistance to endocrine therapy and chemotherapy of breast cancer patients correlates with elevated CHAF1A expression as indicated by Kaplan-Meier Plotter. 
Breast cancer patients with elevated expression of CHAF1A showed shorter time to develop disease recurrence when they received $(A)$ chemotherapy and endocrine therapy, $(B)$ chemotherapy treatment, and (C) endocrine therapy treatment as found by Kaplan-Meier Plotter analysis.

\section{Supplementary Files}

This is a list of supplementary files associated with this preprint. Click to download.

- SupplementaryTablesCHAF1A.docx 